tion more faithfully over the whole $x$-range. However, the Hastings approximation achieves, with its four arbitrary constants, a better fit in the range of low, positive $x$-values most often of interest, and it is therefore preferable for most applications. For $x>2$, the Hastings approximation does not fit as well as $P(x)$. This leads to a somewhat paradoxical observation: While the greatest absolute error for $F(x)$ estimated from the Hastings approximation is only about one-fifth that obtained with $P(x)$, the greatest relative error with the latter is two orders of magnitude below those encountered with the Hastings approximation.

University of California

Lawrence Radiation Laboratory

Bio-Medical Research I)ivision

Livermore, California

1. Tables of Normal Probability Functions, NBS, Applied Mathematics Series, No. 23, U.S. Government Printing Office, Washington, D. C., 1963.

2. M. R. SAMPFORD, "Some inequalities on Mill's ratio and related functions," Ann. Math. Statistics, v. 24, 1953, pp. 130-132. MR 14, 995.

3. R. F. TATE, "On a double inequality of the normal distribution," Ann. Math. Statistics, v. 24, 1953, p. 132-134. MR 14, 995.

4. R. G. HART, "A formula for the approximation of definite integrals of the normal distribution function," MTAC, v. 11, 1957, p. 265. MR 19, 1199.

5. C. Hastings, A pproximations for Digital Computers, Princeton Univ. Press, Princeton, N. J., 1955, p. 167. MR 16, 963.

\title{
Rational Approximations to the Solution of the Second Order Riccati Equation*
}

\author{
By Wyman Fair and Yudell L. Luke
}

I. Introduction. In a previous work Merkes and Scott [1] constructed continued fraction solutions to the first order Riccati equation by using a sequence of linear fractional transformations. Fair [2] utilized the $\tau$-method, see the paper by Luke [3], to develop main diagonal Padé approximations to the solution of the first order Riccati equation with rational coefficients. Rational approximations are advantageous to study the behavior of the solutions in a global sense. That is, they are useful for evaluation of functional values in the complex plane including zeros and poles.

In this paper we develop continued fraction (and hence rational) approximations to the solution of a second order nonlinear equation which includes as special cases the equations treated in [1] and [2]. These approximations are obtained by using a sequence of linear transformations which leave the differential equation invariant, see Davis [4], and are presented in Section II. For an application, in Section III, the algorithm is applied to obtain approximations to Painlevé's first and second transcendents.

II. Development of the Rational Approximations. Consider the generalized second order Riccati equation

\footnotetext{
Received May 16, 1966.

* This work was supported by the National Aeronautics and Space Administration under Contract NASA Hq. R\&D 80X0108(64), 10-74-740-124-08-06-11, PR10-2487.
} 


$$
\begin{aligned}
\left(A_{0}+B_{0} y\right) y^{\prime \prime}+\left(C_{0}+D_{0} y\right) y^{\prime}-2 B_{0}\left(y^{\prime}\right)^{2}+E_{0}+F_{0} y+G_{0} y^{2}+H_{0} y^{3} & =0 \\
y(0) & =\beta_{0},
\end{aligned}
$$

where the coefficients in (1) have Taylor's series expansions of the form

$$
\begin{aligned}
& A_{0}=x^{2} \sum a_{k} x^{k}, \quad B_{0}=x^{2} \sum b_{k} x^{k}, \quad C_{0}=x \sum c_{k} x^{k}, \\
& D_{0}=x \sum d_{k} x^{k}, \quad E_{0}=\sum e_{k} x^{k}, \quad e_{0} \neq 0, \quad F_{0}=\sum f_{k} x^{k}, \quad f_{0} \neq 0, \\
& G_{0}=x \sum g_{k} x^{k} \quad \text { and } \quad H_{0}=x \sum h_{k} x^{k},
\end{aligned}
$$

taking all the sums from $k=0$ to $k=\infty$. We further assume that the solution of (1) has a power series expansion of the form

$$
y=\sum_{k=0}^{\infty} \beta_{k} x^{k}
$$

Note that (2) and (3) together with (1) uniquely determine $\beta_{0}$ and $\beta_{1}$. We also require that the coefficients in (3) have the property that the determinants

$$
\Delta_{p}=\left|v_{i j}\right| \neq 0, \quad v_{i j}=\beta_{i+j} ; \quad i, j=0,1, \cdots, p ; p=0,1,2, \cdots,
$$

and

$$
\Gamma_{2 p+1}=\left|u_{i j}\right| \neq 0, \quad u_{i j}=\beta_{i+j-1} ; \quad i, j=1,2,3, \cdots, p ; p=1,2,3, \cdots .
$$

Then (4) insures that $y$ has a continued fraction expansion of the form

$$
y=\frac{\alpha_{0}}{1}+\frac{\alpha_{1} x}{1}+\frac{\alpha_{2} x}{1}+\cdots, \quad \alpha_{0}=\beta_{0} .
$$

A transformation of the type

$$
y=\frac{m(x)+n(x) y^{*}}{p(x)+q(x) y^{*}}
$$

where $m, n, p$ and $q$ are polynomials in $x$ may be necessary to bring the differential equation into the required form. We suppose that this has already been done. See [4] for the results of applying transformations of this type to (1). We give an example in Section III.

The even approximants of $(\tilde{j})$ are the main diagonal Padé approximations to $y$ which have the following properties. Let

$$
y_{n}=\frac{P_{n}}{Q_{n}}=\sum_{k=0}^{n} p_{n, k} x^{k} / \sum_{k=0}^{n} q_{n, k} x^{k}
$$

be the $n$th order main diagonal Padé approximation to $y$. If $Q_{n}$ is formally divided into $P_{n}$, the resulting power series agrees with the power series representation of $y$ for the first $(2 n+1)$ terms. The polynomials $P_{n}$ and $Q_{n}$ both satisfy the recurrence relation

$$
\begin{aligned}
& P_{n}=\left[1+\left(\alpha_{2 n-1}+\alpha_{2 n}\right) x\right] P_{n-1}-\alpha_{2 n-1} \alpha_{2 n-2} x^{2} P_{n-2}, \\
& P_{0}=\alpha_{0}, \quad P_{1}=\alpha_{0}\left(1+\alpha_{2} x\right), \quad Q_{0}=1 \text { and } Q_{1}=1+\left(\alpha_{1}+\alpha_{2}\right) x .
\end{aligned}
$$

Thus, rational approximations to the solution of (1) are immediately forthcoming if the values $\alpha_{1}, \alpha_{2} \cdots$ ('an be computed. These values can be obtained by utilizing 
a sequence of linear fractional transformations. Let

$$
y=y_{0}, \quad y_{n}=\alpha_{n}\left(1+x y_{n+1}\right)^{-1}, \quad n \geqq 0 .
$$

Repeated application of (8) to (1) and division by $\alpha_{n} x$ at each step yields

$$
\begin{aligned}
\left(A_{n+1}+B_{n+1} y_{n+1}\right) y_{n+1}^{\prime \prime}+ & \left(C_{n+1}+D_{n+1} y_{n+1}\right) y_{n+1}^{\prime}-2 B_{n+1}\left(y_{n+1}^{\prime}\right)^{2} \\
& +E_{n+1}+F_{n+1} y_{n+1}+G_{n+1} y_{n+1}^{2}+H_{n+1} y_{n+1}^{3}=0,
\end{aligned}
$$

where

$$
\begin{aligned}
& A_{n+1}=-A_{n}-\alpha_{n} B_{n}, \\
& B_{n+1}=-x A_{n}, \\
& C_{n+1}=-2 x^{-1}\left(A_{n}+\alpha_{n} B_{n}\right)-C_{n}-\alpha_{n} D_{n}, \\
& D_{n+1}=2 A_{n}-x C_{n}, \\
& E_{n+1}=x^{-1}\left(\alpha_{n}^{-1} E_{n}+F_{n}+\alpha_{n} G_{n}+\alpha_{n}^{2} H_{n}\right), \\
& F_{n+1}=-x^{-1}\left(C_{n}+\alpha_{n} D_{n}\right)+3{\alpha_{n}}^{-1} E_{n}+2 F_{n}+\alpha_{n} G_{n}, \\
& G_{n+1}=2 x^{-1} A_{n}-C_{n}+3 \alpha_{n}^{-1} x E_{n}+x F_{n},
\end{aligned}
$$

and

$$
H_{n+1}=\alpha_{n}{ }^{-1} x^{2} E_{n}, \quad n=0,1,2, \cdots
$$

It is easily shown that

$$
A_{n+1}(0)=B_{n+1}(0)=C_{n+1}(0)=D_{n+1}(0)=G_{n+1}(0)=H_{n+1}(0)=0,
$$

$E_{n+1}$ and $F_{n+1}$ are defined at $x=0$ and

$$
y_{n+1}(0)=\alpha_{n+1}=-E_{n+1}(0) / F_{n+1}(0), \quad n=0,1,2, \cdots .
$$

Thus the values $\alpha_{k}$ appearing in (5) can be computed recursively.

In the general situation closed form expressions for the $\alpha_{k}$ are not known. Details concerning their asymptotic values are lacking. If such data were available, the question of convergence of the approximations could be settled by application of known theorems in the theory of continued fractions. See, for example, Wall [6] and Perron [7]. In numerous special cases convergence has been established, see the latter two references, Merkes and Scott [1], Fair [2] and Luke [3]. Heuristic evidence indicates convergence for rather liberal conditions on the coefficients of the differential equation (1) and a wide range of the variable. In this connection one can imply convergence of the approximants (6) by comparing the values of the $n$th and the $(n+1)$ st approximations. In practice this works very well, and in the cases investigated the actual error of the $n$th approximant is the order of magnitude of the difference $y_{n+1}(x)-y_{n}(x)$.

III. Examples. We consider two examples which exhibit the utility of these approximations when used to approximate both the function and its poles. tions

Painleve's first and second transcendents are defined by the differential equa-

$$
u^{\prime \prime}-6 u^{2}-\lambda x=0, \quad u(0)=1, \quad u^{\prime}(0)=0,
$$


TABLE I

\begin{tabular}{c|c|r}
\hline$x$ & $u(x)$ & \multicolumn{1}{c|}{$u_{6}(x)$} \\
\cline { 3 - 3 } 0.0 & 1.0000 & 1.0000 \\
0.1 & 1.0305 & 1.0305 \\
0.2 & 1.1264 & 1.1264 \\
0.3 & 1.3015 & 1.3015 \\
0.4 & 1.5831 & 1.5831 \\
0.5 & 2.0228 & 2.0228 \\
0.6 & 2.7212 & 2.7212 \\
0.7 & 3.8909 & 3.8909 \\
0.8 & 6.0383 & 6.0383 \\
0.9 & 10.6226 & 10.6223 \\
1.0 & 23.3936 & 23.3860 \\
1.1 & 87.7732 & 87.3769 \\
\hline
\end{tabular}

TABLE II

\begin{tabular}{c|c|c}
\hline$x$ & $v(x)$ & $v_{6}(x)$ \\
\cline { 2 - 3 } 0.0 & 1.0000 & 1.0000 \\
0.1 & 1.0152 & 1.0152 \\
0.2 & 1.0626 & 1.0626 \\
0.3 & 1.1464 & 1.1464 \\
0.4 & 1.2742 & 1.2742 \\
0.5 & 1.4592 & 1.4592 \\
0.6 & 1.7254 & 1.7254 \\
0.7 & 2.1184 & 2.1184 \\
0.8 & 2.7369 & 2.7369 \\
0.9 & 3.8344 & 3.8343 \\
1.0 & 6.3110 & 6.3104 \\
& & \\
\hline
\end{tabular}

and

$$
v^{\prime \prime}-2 v^{3}-x v-\mu=0, \quad v(0)=1, \quad v^{\prime}(0)=0,
$$

respectively. In what follows, $\lambda=\mu=1.0$.

To cast (12) and (13) into the required form of (1), we set $u=1+3 x^{2} \bar{u}$ and $v=1+1.5 x^{2} \bar{v}$ in which case (12) and (13) become

$$
3 x^{2} \bar{u}^{\prime \prime}+12 x \bar{u}^{\prime}+\left(6-36 x^{2}\right) \bar{u}-54 x^{4} \bar{u}^{2}-(6+x)=0, \quad \bar{u}(0)=1
$$

and

$$
\begin{gathered}
3 x^{2} \bar{v}^{\prime \prime}+10 x \bar{v}^{\prime}+\left(5-18 x^{2}-6 x^{3}\right) \bar{v}-27 x^{4} \bar{v}^{2}-13.5 x^{6} \bar{v}^{3}-(5+4 x)=0, \\
\bar{v}(0)=1 .
\end{gathered}
$$

Now $u$ has a pole of the second order at $x=1.2067$ and $v$ has a simple pole at $x=1.1577$. This behavior manifests itself in Tables I and II above in which $\bar{u}_{6}$ and $\bar{v}_{6}$ are the sixth order main diagonal Padé approximations to $\bar{u}$ and $\bar{v}$ obtained using the algorithm of $\S \mathrm{II}$. We have

$$
u_{6}=1+3 x^{2} \bar{u}_{6} \text { and } v_{6}=1+1.5 x^{2} \bar{v}_{6} .
$$

The values of $u(x)$ and $v(x)$ were taken from a paper by Simon [5] who used (12) and (13) as examples in a study of a numerical integration technique for the solution of initial value problems in ordinary differential equations.

The poles of smallest magnitude of $u_{6}$ and $v_{6}$ are 1.2058 \pm 1.0134 and 1.1578, respectively, so that one would expect that the poles of $u$ and $v$ could be computed to any desired degree of accuracy using higher order approximations. Indeed this is the case when the approximations converge.

Acknowledgment. The authors take this opportunity to thank Mrs. Geraldine Coombs, Mr. John Nelson and Miss Rosemary Moran for their assistance with the calculations. 
1. E. P. Merkes \& W. T. Scott, "Continued fraction solutions of the Riccati equation," J. Math. Anal. Appl., v. 4, 1962, pp. 309-327. MR 25 \#4167.

2. W. FaIr, "Padé Approximations to the Solution of the Riccati Equation," Math. Comp., v. 18, 1964, pp. 627-634. MR 29 \#6630.

3. Y. L. Luke, "The Padé table and the r-method," J. Math. and Phys., v. 37, 1958, pp. 110-127. MR 20 \#5558.

4. H. T. DAvis, Introduction to Nonlinear Differential and Integral Equations, Chapter 8, Jover, New York, 1962.

5. W. E. Simon, "Numerical technique for solution and error estimate for the initial value problem," Math. Comp., v. 18, 1965, pp. 387-393.

6. H. S. WALL, Analytic Theory of Continued Fractions, Van Nostrand, New York, 1948. MR 10, 32. 25.

7. O. Perron, Die Lehre von den Kettenbrüchen, Vol. II, Teubner, Stuttgart, 1957. MR 19,

\section{On the Evaluation of the Incomplete Gamma Function}

\section{By Roy Takenaga}

0. Abstract. The accurate evaluation of the $\chi^{2}$ distribution for high degrees of freedom by the usual methods is very difficult (even with a digital computer) because the series to be evaluated would become unbearably long. Also, when a series becomes long, more precision in the numbers used is required in order to offset the effects of round-off errors. On a computer this would mean the use of multiple precision. Accurate tables can be, and have been, prepared by use of the Cornish-Fisher approximation. Comparison of the table values with the values obtained by the method in the writer's paper show that these tables have an accuracy of about six significant figures. For practical purposes there seems to be no lack of $\chi^{2}$ tables for high degrees of freedom. The method in the writer's paper is still useful in checking on the accuracy of tables computed by approximate methods or in producing tables with more significant figures. With single precision it can produce tables of seven figure accuracy at a speed far better than could be by the usual accurate methods. Some unique and useful tables can be produced using this method.

1. Introduction. The incomplete gamma function is defined by K. Pearson [2, p. v] to be

$$
\Gamma_{y}(p+1)=\int_{0}^{y} t^{p} e^{-t} d t, \quad-1<p, 0 \leqq y .
$$

This paper presents a method of evaluating the ratio

$$
F_{p}(y)=\Gamma_{y}(p+1) / \Gamma(p+1), \quad-1<p, 0 \leqq y,
$$

for cases in which $p$ is greater than about 1j. For the lower values of $p$ there are a number of integration methods. Among them is the formula [2, p. xv]

$$
\begin{aligned}
F_{p}(y)=\frac{e^{-y} \cdot y^{p+1}}{\Gamma(p+2)}\{1+ & \frac{y}{p+2}+\frac{y^{2}}{(p+2)(p+3)} \\
& \left.+\cdots+\frac{y^{k-1}}{(p+2)(p+3) \cdots(p+k)}+\cdots\right\} .
\end{aligned}
$$

Received January 24, 1966. 